have her motions carefully disinfected, to disinfect bedclothes and underclothes, to wash her hands with an antiseptic solution, and to keep away from the kitchen premises.

IT is with deep regret that we have to announce the death of Dr. James Grey Glover which occurred quite suddenly on Oct. 14th. We hope to publish an extended notice of our old friend and colleague in a future issue.

THE third scientific report of the investigations of the Imperial Cancer Research Fund has been issued in the name of the managers by Dr. E. F. Bashford, general superintendent of the laboratory. We shall deal at length with the report in a future issue.

AT a special session of the justices of the city of Liverpool, held on Oct. 6th, Mr. J. E. O'Sullivan, J.P., L.R.C.P. \& S. Edin., was appointed one of the judicial authority under the Lunacy Act, 1890.

\section{AN ACCOUNT OF THE POST OFFICE SANATORIUM MOVEMENT.'}

By Chas. H. GarlaAND,

SECRETARY AND FOUNDER OF THE POST OFFICE SAYATORIUM SOCIETY CHAIRMAN OF THE NATIONAL ASSOCIATION FOR THE ESTABISISMENT AND MAINTENANCE OF SANATORIA FOR WORKERS ; MEMBER OF THE COUNCIL OF THE NATIONAL ASSOCIATION FOR THE PREVENTION OF CONSUMPTION MEMBER OF THE BRTTISH EXECUTIVE OF THE INTERNATIONAX CONGRESS ON TUBERGULOSIS (WASHIYGTON, 1908), ETC.

THE Post Office Branch of the National Association for the Establishment and Maintenance of Sanatoria for Workers Suffering from Tuberculosis was registered on Jan. 16th, 1907, and the first of its patients was admitted to Benenden Sanatorium on March 4th, 1907. It is a voluntary association and came into existence as the result of a conference held on Sept. 29th, 1906. But prior to this, of course, much propaganda work was done among the employees who now form its membership. The method of this organisation cannot fail to be of interest, and I will therefore preface this brief account of its working with some account of its formation.

For years the belief existed among post office employees that consumption demanded an excessive number of victims in the postal service, and this belief is common among post office workers in other European countries. The support of this belief is the undoubted fact that tuberculosis does account in the British post office service for nearly half of the total mortality. This result is due largely to exclusion of other diseases by medical selection and inspection. The belief supplied some of the groundwork on which the Post office Sanatorium Society was eventually erected. ${ }^{2}$ In January, 1902, after many less widely circulated articles and papers had been written, 25,000 copies of a pamphlet ${ }^{3}$ were printed and distributed by the agency of the post office labour societies. By these means a lively interest in the subject was created and the ground prepared for the launching of the scheme for the provision of sanatorium treatment. On March 6th, 1903, a resolution was moved by myself at the annual conference of the Postal Telegraph Clerks' Association of Great Britain held at Leeds, as a result of which a subcommittee was appointed to consider the practicability of providing sanatorium treatment for post office servants, and power was given to them to coöperate with other societies in futherance of this object. In May of the same year, under the auspices of the Hospital

"A paper entitled "An Account of the Organisation and Working of the Post Office Branch of the National Association for the Establishment and Maintenance of Sanatoria for Workers Suffering from Tuberculosis, known as the Post Office Sanatorium Society," contributed to the International Congress on Tuberculosis at Washington.

2 Vide article by Dr. T. D. Lister and C. H. Garland, British Journal of Tuberculosis, July, 1908

3 Consumption in the Post Office with Some Hints on Prevention, by C. H. Garland (the first part reprinted from Trre LANCEI of Sept. 14th,
Saturday Fund, an effort was commenced to form a combination of trade unions, friendly societies, and other corporations for the purpose of erecting self-maintaining sanatoriums for workers. A consultation between the secretaries of the two movements resulted in a combination which has been mutually fruitful, and has been cemented by the fact that the writer has acted as chairman of the combined movements and secretary of the Post Office Society during the initial stages. The post office subcommittee called a conference at the General Post Office, London, on August 27th, 1904, at which a provisional committee was elected representing all arms of the post office service. As a result of the work of this committee a circular was drawn up and nearly 100,000 copies were issued to postmasters and heads of departments throughout the United Kingdom, with a request that it should be distributed to the members of their staffs. The chief points of the scheme proposed as set out in the circular may thus be summarised.

By the aid of the National Association (then called the National Committee) for the Establishment and Maintenance of Sanatoria for Workers Suffering from Tuberculosis it was possible for workers' organisations to obtain beds in sana. toriums for an inclusive charge of $£ 65$ per year. It had been estimated that the number of "first year cases" among post-office employees which would be likely to prove suitable for treatment would not exceed 2 per 1000 living among the members of the projected society. A subscription of $2 s$. per year, or less than $\frac{1}{2} d$. per week, would raise $\$ 100$ per year per 1000 members, and as each bed would provide on the average for three patients per year the amount raised would provide for all cases likely to arise, pay management expenses, and possibly meet other charges. The response of the post-office employees of all grades, of whom there are nearly 200,000, was enthusiastic, and an estimate of prospective membership given to the inaugural conference of Sept. 29th, 1906, set the number at 32,000. This has been exceeded, and is daily increasing, and on May 15th, 1908 , it stood at 34,500 . At the inaugural conference a set of rules was adopted and a committee and officers were appointed. The rules of the society were registered at the Registry of Friendly Societies on Jan. 16th, 1907. The first patient was sent to Benenden Sanatorium on March 4th 1907, and up to May 15th, 1908, 108 cases had been dealt with. Table I. shows the number of cases, the post office rank, the office in which he or she is employed, and the dates of admission and discharge, and Table II. shows the results obtained.

\section{TABLE II.-Summary of Results.}

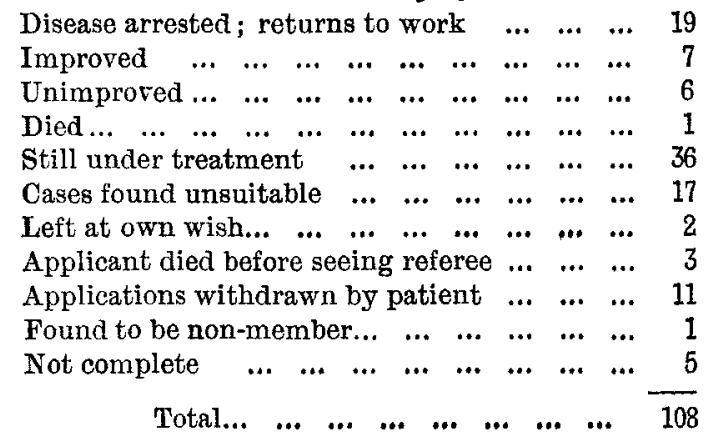

Completed cases. - From the accompanying tables it will be seen that the treatment of 33 cases has been completed at the sanatorium and 36 are still under treatment. Of the 33 cases completed 19 left with the disease arrested and returned to work, seven left improved, six unimproved, and one died at the sanatorium. Of these one case of arrest has relapsed and one unimproved case has been readmitted to the sanatorium. The average length of treatment has been 16 weeks and a day. The longest stay was 41 weeks and the shortest four weeks.

Membership. - The total paid-up membership is shown by the balance sheets to have been in January, 1907, 31,915; July, 1907, 32,641; and May 15th, 1908 (partly estimated), 34,500. At the time of writing (July 31st) the membership stands at 35,350.4 The number of branches is 616 .

Collection of funds. - The method of collection is exceedingly simple and, so far as the society is concerned, exceedingly cheap. The Postmaster-General agreed to deduct the sum of $1 s$. half-yearly from the salary or wages of any of his officers who gave consent to such deduction. 
TABLE I.-Shoning Cases Dealt With up to May 15th, 1908.

\begin{tabular}{|c|c|c|c|c|c|}
\hline No. & Rank. & Office. & Admitted. & Discharged. & Duration. \\
\hline 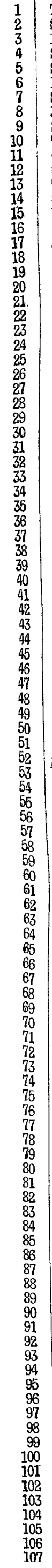 & 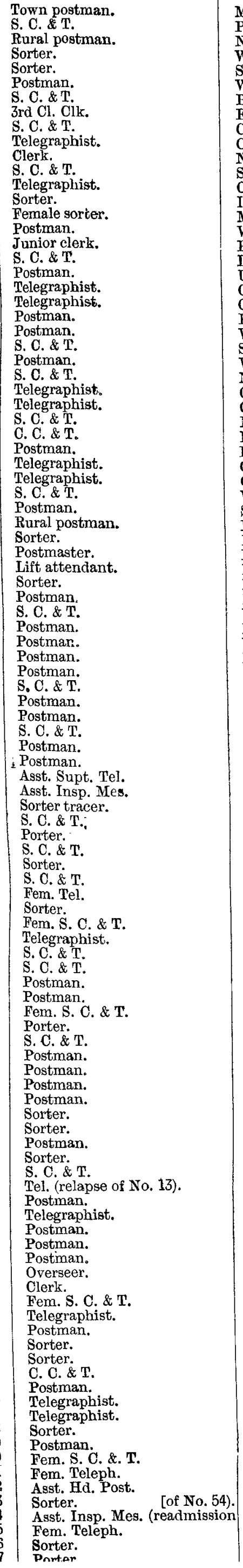 & 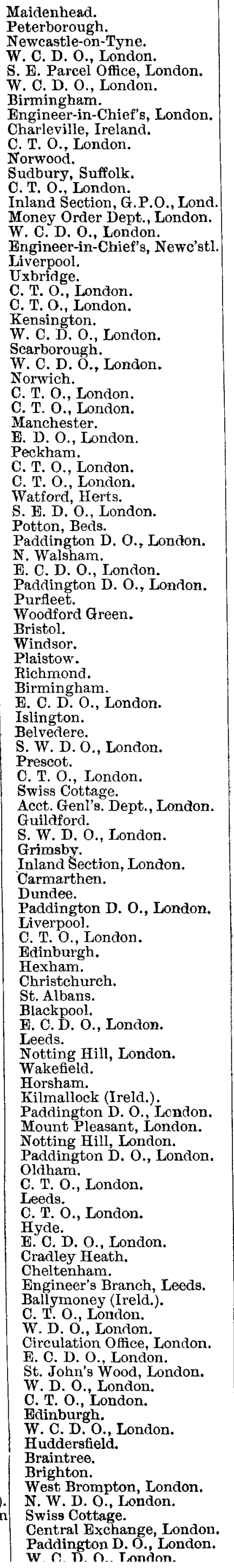 & 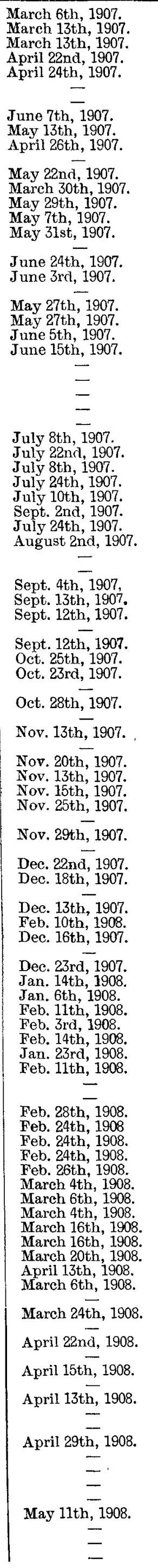 & 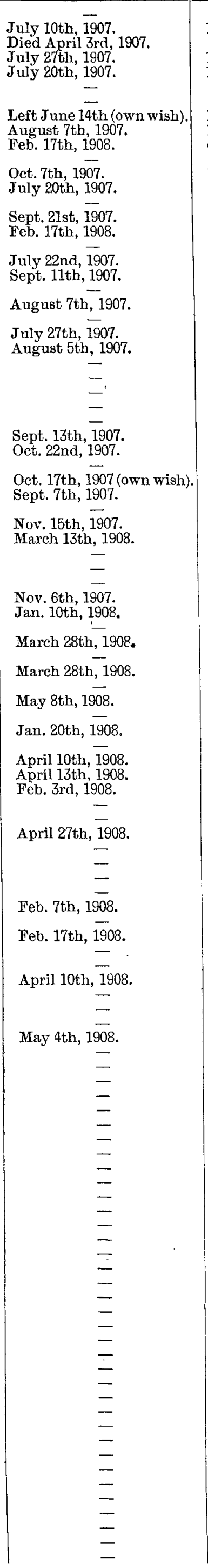 & 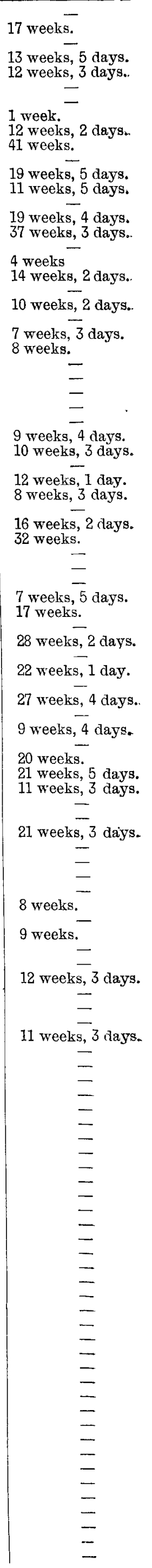 \\
\hline
\end{tabular}


The consent is contained in the form of admission, the lower half of which is sent to the postmaster of the town to which the member is attached when the membership commences. The money having been deducted and brought to account at the headquarters of the Comptroller and Accountant-General in London, is then paid in bulk by warrant to the treasurer of the society. This method of collection insures regularity of contribution, avoids arrears, and gives the full value of the subscription to the society. There is little doubt that the collection of subscriptions by the Postmaster-General, which was undertaken at my suggestion, has gone far towards insuring the success of the society and keeping down the cost of administration. It forms, indeed, a very valuable contribution to the movement by the Post Office Department, and may be reckoned as a species of State aid.

Propaganda and recruitment of new members.-For the purpose of disseminating information concerning the society and assisting in recruitment, show-cards are, by consent of the Postmaster-General, hung in the retiring rooms and waiting-rooms used by the staffs at the post offices throughout the kingdom. Over 2000 of these cards have been issued. Local committees are encouraged for the purposes of organisation, and the literature of the National Association is used for purposes of propaganda. In addition, a circular letter is supplied to local secretaries for the purpose of appeals to non-members. It is intended to bring this up to date from time to time.

Medical papers and routine of treatment.-A member qualified for benefit under the Rules (Rule III., secs. 1 to 8), having reason to believe that he is suffering from the symptoms of early pulmonary tuberculosis, applies to the secretary for the necessary forms of certificate, \&c. He is supplied with papers, accompanied by a circular letter of instructions. The papers, after being filled up, are returned to the secretary of the society, and the certificate form is forwarded by him to the secretary of the sanatorium accom. panied by a certificate sesting out that the applicant is a member of the affiliated society. The patient is then referred to the nearest medical referee of the sanatorium who fills in a further certificate form. Should the case be considered unsuitable by the referee, the patient is informed and the routine ends. Should he, however, be suitable, the referee's certificate is returned to the secretary of the society. A copy is made and attached to the papers and accompanied by a covering letter is forwarded to the Secretary of the General Post Office. On the consideration of these papers leave is granted for a period of about from four to six weeks and the patient proceeds to the sanatorium, his travelling expenses being paid by the society. At the end of that period a further supplementary medical report is filled in by the medical superintendent of the sanatorium and forwarded to the Secretary, General Post Office. On this report further leave is granted and the series of reports is continued at intervals until the patient's discharge. This discharge is entirely in the discretion of the sanatorium authorities; the society, being desirous that the best medical results should be obtained, sets no limit to the stay, and does not in any way interfere with the medical discretion. Post Office medical officers-numbering over 2000 -in all parts of the country have been instructed in the class of case likely to prove suitable by the issue to them of a circular letter in which is set out, inter alia, the desirability of seeking among their catarrhal and dyspeptic patients for the signs of incipient phthisis. By this means and by the publication of the "Symptoms of Early Consumption of the Lungs" in the Rule Book and on the show cards it is sought to get the cases "before they are too far advanced for successful treatment. The result of these efforts has been that we have obtained our patients far earlier in the course of their disease than those received from societies not adopting similar means. The friends of patients have been able to detect early symptoms in the sufferers and have urged them to seek advice.

Support of patient's family during treatment. - The whole of the patient's salary is paid by the Post Office Department for six months and the half during a further six months. This money goes in its entirety to support his family. No charge is made upon him for his treatment at the sanatorium, and his railway expenses are paid on admission and discharge (Rule III., sec. 7). By this means all source of worry is removed from the patient and his family, and the usual reason for incomplete treatment is avoided.
Record of membership. - In consequence of the method if collection the record of membership is very simple. The name, rank, office, and date of joining the society are in the case of each member entered in a ledger under the name of the office. Each half year new entrants and alterations are notified to each postmaster on a special form. ${ }^{6}$ Postmasters not receiving such a notice of addition to membership employ another form for the purpose of notifying any alterations due to transfers, resignations, deaths, \&c. These alterations are entered in the ledgers and the record thus brought up to date.

Voluntary contributions to building fund.-The above organisation represents the means of maintaining beds. But the erection of Benenden Sanatorium has been by voluntars contribution, and in this contribution participating societies have been invited to share. The Post Office Society has subscribed over $£ 650$ in this way by voluntary contributions of small sums by its members. Fines inflicted on the post office staff for late attendance are, by permission of the Postmaster-General and with the consent of the staff, allocated for the purpose of the building fund.

General considerations. - The Post Office Society, by its methods and success, has been the chief support of the National Association during the initial stages of its work. The post office staff, comprising men and women of all grades of society, and scattered throughout the country, has been an excellent means of propagating the ideas and know. ledge necessary as forerunning the practical work of cure and prevention of tuberculosis. Local committees have been formed and have acted as missionary centres whose work has reached far outside post office circles. The society itself stands as an example of what working men and women can do by self-help and of how it can be done and at what cost. In its capacity as exemplar it has had a tremendous effect in stimulating interest and encouraging application. The intelligent interest of the post office staff has facilitated the distribution of literature to the best advantage, and if the movement for self-help against tuberculosis among the workers meets with its deserved and expected success it will owe very much of that success to the example of the Post Office Sanatorium Society and to the men and women who have come from the ranks of the post office movement to aid the progress of the larger work.

\section{APPENDIX A.}

The following are the "symptoms" referred to in the text. They form page 4 of the book of rules of the Post Office Sanatorium Society :-

The Symptoms of Early Consumption of the Lungs.

A hint to those who may be suitable patients for the sanatorium Application must be made in the earliest stages, and not when already ill and weak. Chronic or advanced cases cannot be retained or received in a sanatorium.

Indigestion, very gradual loss of weight and strength, slight Indigestion, very gradual loss of weight and strength, slight
pains about the shoulders and back, cough at first dry and tickling pains about the shoulders and back, cough at first dry and tickling occasional sweating at night or on slight exertion, shortness breath and palpitation. and a pale complexion becoming flashed in the afternoon and evening, are symptoms which, taken together,

demand a thorough examination of the chest stripped to the waist.
A history of blood-spitting or of pleurisy in such a case is a strong A history of blood-spitting or of ple

additional reason for examination. for Consumption, IIampstead and Northwood, and honorary advisory physician to the Council of the National Association for the Establishment and Maintenance of Sanatoria for Workers suffering from Tuberculosis.

The following is an abstract from the show cards exhibited in the retiring rooms of post offices throughout the United Kingdom :-

The Symptoms of Early Consumption of the Lungs : Hints to those who may be Suitable Patients for the Sanatorium.

Persons suffering from indigestion, loss of weight and strength night sweats, cough, with or without blood-spitting, should at once be thoroughly examined by a doctor.

\section{APPENDIX $B$.}

The following is a copy of a circular letter issued to post office medical officers:-

\section{7, Elm Bank Gardens, Barnes, S.W.} January, 1908

Dear Sir,-With the permission of the Postmaster-General I am DEAR SIR, - With the permission of the Postmaster-General I am
sending you under the same cover a copy of the rules of the Post Office sending you under the same cover a copy of the rules of the Post Office
Sanatorium Society, from which you will be able to obtain particularg Sanatorium society, from which
of its organisation and methods.

The society is in no sense official, although its membership is entirel
The composed of post office employees, but is a purely voluntary effort to

5 These forms are supplied by the society but are stocked by the stores department of the General Post Office and issued with the ordinary official stationery. 
deal with cases of early consumption among its members. As some 34,000 persons of either sex have been enrolled it is probable that some of the patients who come to you for advice may be members. It will type of case which it is you to know the working

tye sanatorium at Benenden (Kent), to which as many as possible of our cases are sent, is devised only for the earliest stages of the disease, is intended to be largely educational, and is quite unsuitable for very acute cases, or for chronic or advanced and or for rapidly advancing cases The institution has sufficient staff and facilities to deal with early cases The institution has sufficient staff and facilities to deal with early cases, small percentage of complications that occur in such well-selected early cases.

As general rules for the selection of cases the Council of the sanatrium is advised to suggest that :-

(1) Cases which have existed for more than six months from the outset (dry cough and indigestion and lassitude), and still more so, cases which have been unable to work for more than a few weeks, are likely to be unsuitable for sending to the medical referees.

(2) Cases which present any evidence of the disease in more than one lobe of the lung require very careful selection.

(3) Cases presenting disease in more than two lobes, or complicated by other affections (e.g., diabetes, Bright's disease, chronic bronchitis, alcoholism), are unsuitable for sending to the referees.

(4) Cases which have previously undergone arrest after an attack involving only a limited area of lung, and have remained in good health for a considerable time (i.e., not immediate relapse on return from an institution) and are otherwise suitable (as above suggested) should be sent to the referees.

From motives of benevolence a few advanced cases have been sent to the institution in error, one arriving moribund after a journey of over 200 miles. Unsuitable cases can only suffer by being sent to such a sanatorium as Benenden, and should be referred to the chest hospitals, or infirmaries, or treated at home. On the other hand, the medical attendants of the postal staffs can greatly assist in this attempt to check the waste due to pulmonary tuberculosis, by seeking out the cases of early tubercle among their dyspeptic and catarrhal patients and advising the patient, if he is a member of the Sanatorium Society, to apply to the secretary of the society with a view to obtaining treatment. The best cases for sanatorium treatment would be detected by the symptoms, by the thermometer and by auscultation, before the expectoration contains tubercle bacilli, or is typical in appearance.

The treatment given by the society can only be given to its members under any circumstances. To these, however, there is absolutely no charge, and if application is made under Rule III., section 7 , all
travelling expenses incurred in visiting the medical referee, and in going to and from the sanatorium, will be paid.

Trusting to receive your coopperation and assistance in the work,

$$
\text { I am, yours faithfully, }
$$

C. H. GarLand, Secretary.

Post Office Branch of the National Association for the

Establishment and Maintenance of Sanatoria for

Workers Suffering from Tuberculosis.

\section{MOTORING NOTES.}

\section{By C. T. W. Hirsch, M.R.C.S. ENG., L.R.C.P. LoNd.}

AN important part of a car, one that seldom gives trouble, though it is as a rule grossly neglected, is the springs which take the strain and weight and intercept vibration. Considering how often the springs of a car are left to look after themselves, the small number that break speaks well for the excellence of the material of which they are made usually and of the manner in which the are forged and tempered. A broken spring may lead to serious trouble and all could be avoided by a little attention. In order that a spring may do its work properly each component plate must work clear and free of its neighbours, but as a result of the entrance of washing or rain-water between the plates one may become rusted and thus set and then it may fracture. All this can be prevented by making a rule, say once every three months, to jack up the car, to loosen the clips that keep the component plates together, to clean them with paraffin oil between each, to wipe them dry, and then to spread a layer of grease between each. When the general overhaul takes place the spring should be taken down, dismembered, and each plate scraped, oiled, and then the whole reassembled. When this is done the shackle pins should receive attention. Many motorists, even some who really take an especial pride in the sweet running of their machinery, would be astonished if they examined these parts, I have often seen pins taken out that were more than threequarters worn through, and I must confess that I once found such a hinge pin on one of my own cars. Until quite recently motor manufacturers merely made little holes for the lubrication of these parts, holes that collected mud, water, and dust, and required cleaning out every time one wanted to put oil in. Now I am glad to say that proper lubricators seem as a rule to be fitted, and even then they are difficult to oil properly, because a shackle pin is subject to a heavy load and the reciprocating motion about it is slight, so there is a tendency for the lubricant to accumulate on the upper side, where the pressure is normally zero and it does not spread over the actual bearing surface, as in the case of a shaft which rotates, so it is as well, in addition to daily oiling them, to take them out occasionally and to see that the oil does not remain on the top and when replacing to grease them all round. Attention to such little matters as these prevents breakdowns and expense. In order that they should not be overlooked it is well to have a "garage" day once a month, and among other points that should then be seen to is the inspection of hinge and shackle pins. I keep two cars and each has one garage day a month, when valves can be, if needful, ground in : all nuts checked and, if required, tightened; all moving parts oiled; sparking plugs cleaned or replaced by new ones ; clutch leather inspected, and if necessary dressed with collan oil or fuller's earth; crank case emptied of oil, cylinders washed with paraffin, dirty paraffin drained, and case filled up to correct level with clean oil, and about once in 12 months the bevel and gear boxes require similar treatment and filling up with fresh grease, and, of course, every month they should be inspected and either grease or oil added. Lubricating pipes are also better for a monthly wash out with paraffin. From chats I have had with colleagues who own cars, seemingly few ever wash out their gear and bevel boxes. It should be remembered that there must always be a certain amount of wear in the bearings, and that thus fine metallic dust finds its way into the grease and oil, and this is anyway not an ideal lubricant for the gears. The procedure is to fill the gear and bevel boxes up with paraffin, and then run the car to and fro; this will reduce the consistency of the lubricant, so that when the plug underneath is taken out the mixture will run out; then the plug should be replaced, and more paraffin added. In the gear box the lid can be removed, and the gears brushed over with an old tooth-brush; and to clean the bevel and differential boxes the back axle can be jacked up and the wheels turned round, so that the grease and paraffin are well mixed before the plug is taken out. After replacing the plug and fllling up with paraffin and again working the parts the dirty paraffin can be run off and fresh grease and oil added. It is important to remember that in cars having a direct drive on the top gear, the first motion shaft is in two parts, that by means of some form of dog clutch is made solid when driving direct on the high speed, but on the other speeds one portion of this shaft revolves in the other, a gun-metal bush being, as a rule, provided. As a result of centrifugal action the grease may not enter this bearing and it is one that is very apt to "fire." In gear boxes with this form of transmission it is well to use a large proportion of oil in the gear box and also occasionally to remove the lid and with an oil squirt inject some lubricant directly into this bearing. In many cars with this type of transmission $I$ have seen this bearing so worn after a few months that a new bush had to be fitted, a job that meant taking down the gear box and which could have been avoided by using thinner oil and occasionally personally lubricating the bearing. It is much less trouble to do that than to have a new bush turned and fitted. A friend of mine has recently fitted to his gear box a simple arrangement that makes it absolutely certain that this bearing gets its dose of oil. To the side of the gear box a strip of spring steel is fixed so that it presses against the oil hole in the first motion shaft ; this hole, of course, pierces the outer sleeve of the shaft and the gun-metal bush and there ends in an oilway; as the shaft revolves, the spring, gently pressing, acts as a sort of pump and also prevents the lubricant that so enters into the bearing from being thrown out, and then, if there is a proper oil-way cut on the inside of the bush, that bearing is bound to be kept greased. As the shaft revolves in grease the pressure of the spring will not cause any friction worth mentioning.

Accumulators are long-suffering parts of a car, and really if they receive the attention they should the trade in these articles would not be brisk. The great thing is to use them and also never fully to discharge them. A good plan is to have three, two always on the car, and to use one one day and the other on the following, and once every three weeks to take out one and replace it by a fully charged one, the taken out one being left at an electric light station for charging and fetched in three weeks, when another can be 